\title{
ARTICLE
}

\section{Structural basis for catalytically restrictive dynamics of a high-energy enzyme state}

Michael Kovermann', Jörgen Ådén ${ }^{1}$, Christin Grundström¹, A. Elisabeth Sauer-Eriksson', Uwe H. Sauer ${ }^{1}$ \& Magnus Wolf-Watz ${ }^{1}$

An emerging paradigm in enzymology is that transient high-energy structural states play crucial roles in enzymatic reaction cycles. Generally, these high-energy or 'invisible' states cannot be studied directly at atomic resolution using existing structural and spectroscopic techniques owing to their low populations or short residence times. Here we report the direct NMR-based detection of the molecular topology and conformational dynamics of a catalytically indispensable high-energy state of an adenylate kinase variant. On the basis of matching energy barriers for conformational dynamics and catalytic turnover, it was found that the enzyme's catalytic activity is governed by its dynamic interconversion between the high-energy state and a ground state structure that was determined by X-ray crystallography. Our results show that it is possible to rationally tune enzymes' conformational dynamics and hence their catalytic power-a key aspect in rational design of enzymes catalysing novel reactions.

\footnotetext{
${ }^{1}$ Department of Chemistry, Chemical Biological Centre, Umeå University, Umeå 90187, Sweden. Correspondence and requests for materials should be addressed to M.W.-W. (email: magnus.wolf-watz@chem.umu.se)
} 


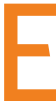
nzymatic catalysis changes the timescales of otherwise slow chemical reactions, making them commensurate with those of biological processes. An enzyme's capacity to enhance the rate of its catalysed reaction depends on its ability to reduce the free energy of transition state compound(s) (that is, formation of Michaelis' complexes), activate functional groups, dehydrate active sites and align substrates in an optimal geometry for reaction. These functionalities are linked to the enzymes' conformational dynamics, which are defined in terms of the timedependent displacement of atomic coordinates. In an enzymatic reaction cycle, it is possible to identify stable ground states that can be characterized experimentally using techniques such as $\mathrm{X}$-ray crystallography and nuclear magnetic resonance (NMR) spectroscopy. Such ground states include substrate-free and substrate-bound states, but high-resolution ground state structures alone cannot fully explain enzymes' catalytic power. Enzymes dynamically access structures with higher free energies than those of the stable ground states. These high-energy states are transient and not sufficiently populated to permit direct spectroscopic observation. Consequently, the dynamic interconversions of enzymes' ground and high-energy states have mainly been characterized indirectly via NMR relaxation dispersion experiments ${ }^{1,2}$, which have been used to unravel the catalytic cycles of cyclophilin A $(\mathrm{CypA})^{3,4}$, adenylate kinase $(\mathrm{AdK})^{5}$, dihydrofolate reductase (DHFR) ${ }^{6}$ and RNase $\mathrm{A}^{7}$, and also to explore the structures of sparsely populated folding intermediates ${ }^{8}$. It has been demonstrated that certain enzymes' rates of catalytic turnover are limited by their conformational dynamics ${ }^{4-6,9}$. AdK catalysis, which plays a vital role in cellular energy homeostasis by catalysing the reversible magnesiumdependent formation of ADP from AMP and ATP ${ }^{10}$, is dependent on distinct ATP- and AMP-binding subdomains (ATPlid and AMPbd; Fig. 1a). It has been suggested that apo AdK exists in a dynamic equilibrium between open- and closed-like conformational states ${ }^{1,12}$, and that certain mutations can modulate this equilibrium ${ }^{13}$. The turnover rate of $\mathrm{AdK}$ is limited by product release, and it has been shown that the microscopic step accountable for this slow release is the re-opening of the substrate-binding subdomains ${ }^{5,14}$ (Fig. 1a). Thus, the conformational dynamics required for AdK catalysis is given by $k_{\text {conf }}$, which is defined as the sum of forward and reverse reaction rates $\left(k_{\text {conf }}=k_{\text {close }}+k_{\text {open }}\right)$. Here we report the direct NMR-driven observation and molecular characterization of a catalytically indispensable high-energy state of AdK. We also provide evidence for an essential coupling between its conformational dynamics and catalytic turnover. Our results show that it is possible to tune catalytically relevant enzyme dynamics, which is essential for the de novo design of enzymes ${ }^{15}$.

\section{Results}

Selection of mutation sites. To directly observe a transient highenergy state in the AdK reaction cycle by NMR spectroscopy, it is essential to slow down the enzyme's conformational dynamics such that the slow exchange limit is approached. Slow exchange on the NMR timescale means that the rate of interconversion $\left(k_{\text {conf }}=k_{\text {close }}+k_{\text {open }}\right)$ between states is well below the difference in their chemical shifts. Under such conditions, discrete peaks corresponding to the different states of the exchanging system can be observed. Conversely, in the fast exchange limit, the exchanging system produces only one averaged peak. We have previously identified alpha helix 9 of the ATPlid domain (Fig. 1a) as a key element in the allosteric modulation of the opening and closing dynamics of AdK, and shown that a single amino-acid replacement is sufficient to shift the equilibrium between the open and closed states $^{13}$. We speculated that replacing various residues in helix 9 of a

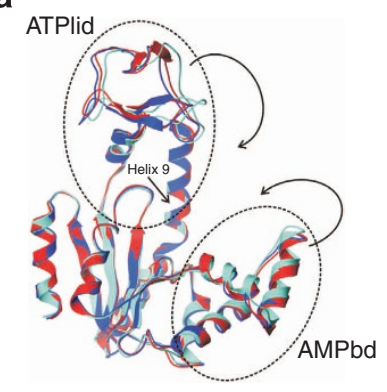

b

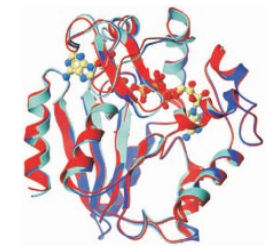

C+S $\underset{k_{\text {off }}}{\stackrel{k_{\text {on }}}{\rightleftarrows}} \mathrm{ES}_{\text {open }} \underset{k_{\text {open }}}{\stackrel{k_{\text {closed }}}{\rightleftarrows}} \mathrm{ES}_{\text {closed }}$
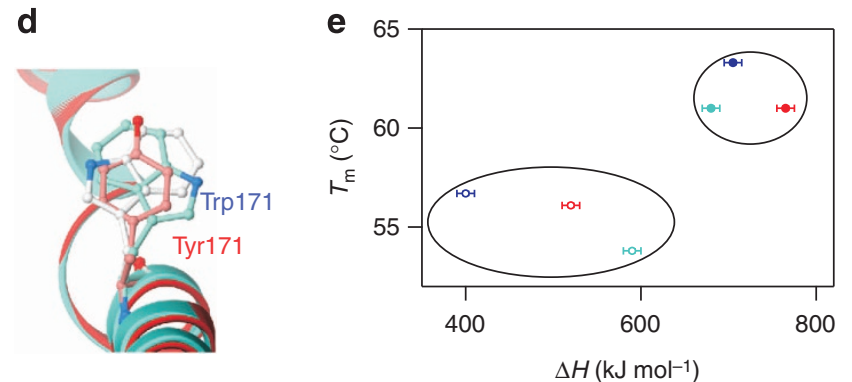

Figure 1 | Ground state structures, energetics and kinetic mechanism of AdK variants. (a) Ribbon diagrams of the crystal structures of the open apo forms of the wild type (4AKE, red), P177A (4X8H, blue) and Y171W (4X8M, cyan) AdK variants. The ATPlid and AMPbd subdomains are labelled and the trajectories for domain closure into the catalytic closed conformation are indicated with arrows. (b) Crystal structures of the closed Ap5a-bound forms of the wild type (1AKE), P177A (4X8L) and Y171W (4X80) AdK variants (colour code as in a). (c) A minimal kinetic mechanism of AdK catalysis indicates the presence of a transient highenergy state (highlighted in red) that is simultaneously open and substrate bound. (d) The side chain of Trp171 in Y171W samples two different conformations (cyan and white) in the Ap5a-bound state (4X8O). The side-chain orientation of Tyr171 for wild type (light red) is shown as reference. Ribbon colours as in $\mathbf{a}$. (e) The global enthalpies of unfolding vary considerably for the apo states (open circles) of the wild type, P177A and Y171W, whereas those of the Ap5a-bound states (closed circles) are more closely clustered. Colour code as in $\mathbf{a}$.

AdK might generate enzyme variants whose conformational dynamics are modulated in a way that would enable the direct detection of functional high-energy states by NMR. We selected two single-point mutations of AdK suitable to investigate this hypothesis. The Prol77 to Ala (P177A) mutation was chosen because proline at position 177 is conserved in $\mathrm{AdK}^{11}$ and introduces a kink in helix 9 whose importance in the enzyme's opening/closing dynamics has been demonstrated by all-atom molecular dynamics (MD) simulations ${ }^{16}$. The Tyr171 to Trp (Y171W) mutation was selected because it was anticipated that this mutation would modulate the enzyme's dynamics and facilitate stopped-flow measurements of the enzyme's ligand binding kinetics.

Characterization of ground state structures. AdK populates two main structural ground states: an open substrate-free state and a closed Ap5a-bound state. P1,P5-di(adenosine- $5^{\prime}$ )pentaphosphate (Ap5a) consists of one ATP and one AMP molecule bridged by a phosphoryl group, and is a good structural mimic of the enzyme's two native substrates. The relevance of this molecule is 
Table 1 | X-ray data collection and refinement statistics.

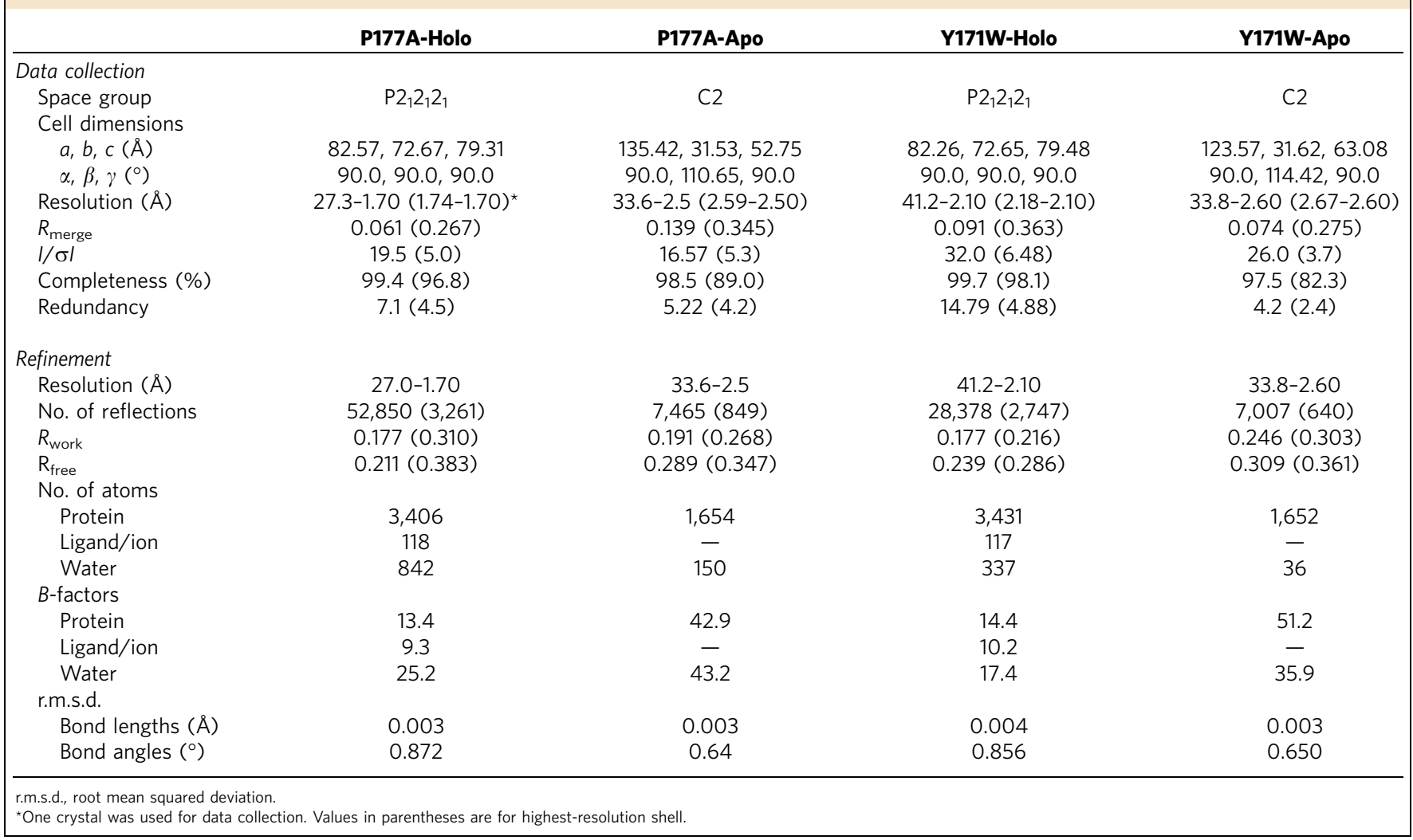

underscored by the fact that the free energy for its binding to $A d K^{17}$ is equal to the sum of the AdK-binding free energies of $\mathrm{AMP}$ and $\mathrm{ATP}^{18}$. The ground state structures of the P177A and Y171W variants in their apo- and Ap5a-bound states were determined by X-ray crystallography (Table 1). The apo and holo structures of both variant enzymes are very similar to that of the wild type (Fig. 1a,b) with only minor differences in the conformations of their AMPbd and ATPlid domains. The catalytic cycles (Fig. 1c) of the two AdK mutants are thus supported by the same underlying structural framework as that of the wild-type enzyme. Notably, the electron density of Trp171 shows that the side-chain samples two rotamer conformations in the Ap5a-bound state (Fig. 1d and Supplementary Fig. 1a,b), and the wild-type Y171 conformation is partially overlapping both of these Trp171 rotamers (Fig. 1d). Analysis of the $C_{\alpha}$ displacements in the X-ray structures of the apo- (Supplementary Fig. 1c) and Ap5a-bound states compared with wild-type AdK (Supplementary Fig. 1d) of the two AdK variants revealed that the displacements of the apo states were significantly more variable than those of the Ap5a-bound states. That is to say, the apo states are more conformationally heterogeneous. NMR chemical shift perturbation analysis also indicated that the conformations of the apo states (Supplementary Fig. 1e,f) differed more than those of the Ap5a-bound states (Supplementary Fig. 1g-i), showing that the apo states exhibit greater structural heterogeneity both in solution and in the crystals. In keeping with these findings, circular dichroism (CD) spectroscopy indicated that the apo states had a broader distribution of thermodynamic unfolding stabilities (Fig. 1e and Supplementary Fig. 1j) than their Ap5abound counterparts (Fig. 1e and Supplementary Fig. 1k).

Ap5a-bound Y171W populates two interconverting structures. Inspection of the proteins' NMR spectra indicated that the
Y171W mutation significantly reduced the rate constant of the enzyme's conformational interconversion $\left(k_{\text {conf }}\right)$ relative to that for the wild type or the P177A mutant (as discussed further below). While no similarly pronounced change in the rate constant was observed for the P177A variant, this mutation is significant because subsequent analyses showed that both, this amino-acid replacement and the Y171W variant, redistribute the native energy landscape of $\mathrm{AdK}$ (see the section $k_{\text {cat }}$ versus $k_{\mathrm{M}}$ and $k_{\text {cat }}$ versus $K_{\mathrm{D}}$ compensation). Most of the expected resonances are visible in the NMR spectra of wild-type AdK in complex with saturating concentrations of the natural substrate ADP (Supplementary Fig. 2a). However, many of these resonances are missing in the NMR spectra of the Y171W mutant in complex with ADP because its conformational interchange proceeds at an intermediate rate on the NMR timescale (Supplementary Fig. 2b). In the intermediate exchange regime, the rate of conformational interconversion is on the order of the difference in chemical shifts between exchanging states, resulting in broadened or missing resonances. This observation suggests that the Y171W mutation slowed down the enzyme's conformational dynamics to the point that it entered the intermediate exchange regime at $25^{\circ} \mathrm{C}$. Further support for this hypothesis comes from the finding that NMR experiments using the Y171W mutant under ADP-saturated conditions at a temperature of $50{ }^{\circ} \mathrm{C}$ yielded spectra closely resembling those for the ADP-saturated wild type at $25^{\circ} \mathrm{C}$ (Supplementary Fig. 2c), indicating that the dynamic properties of the two enzymes are similar at these two temperatures. That is to say, the slowdown of the protein's dynamics induced by the Y171W mutation can be counteracted by raising the temperature. To avoid the problems created by absent resonances in quantitative analysis, we hypothesized that dynamic signatures would also appear in the NMR spectra of AdK variants in complex with the substrate mimic Ap5a (ref. 19). The relevance of using Ap5a when probing conformational 
dynamics is supported by the results of single-molecule fluorescence spectroscopy experiments, which have shown that both substrate (AMPPNP and AMP)-bound ${ }^{14}$ and Ap5a-bound AdK fluctuate between the closed state and less compact structures ${ }^{11}$. When bound to Ap5a, the NMR spectra of the wild type and P177A variant of AdK feature a single set of resonances because the structural interconversions of their bound states are rapid on the NMR timescale. In sharp contrast, the ${ }^{1} \mathrm{H}-{ }^{15} \mathrm{~N}$ heteronuclear single quantum coherence (HSQC) NMR spectrum of Y171W bound to Ap5a shows two sets of resonances at ambient temperature (Fig. 2a and Supplementary Fig. 2d): 61 of its residues give rise to two peaks of roughly equal intensity. These 61 residues are distributed over the entire enzyme (Supplementary Fig. 2d,e), showing that the underlying event is global in nature, and not restricted to the side-chain atoms of Trp171 as seen in the crystal structure (Fig. 1d). It is important to
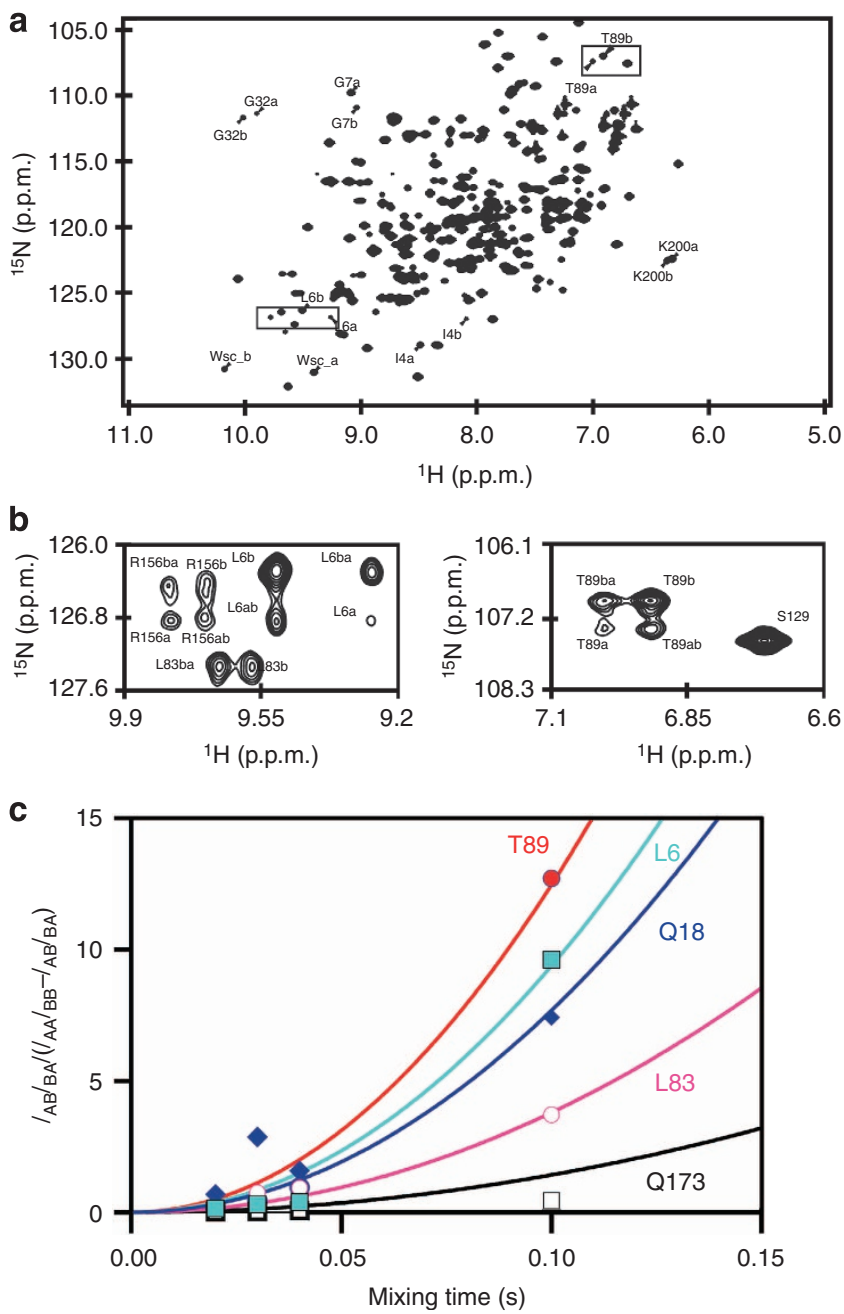

Figure 2 | Observation of exchange kinetics between two Ap5a-bound states of Y171W. (a) Dual peaks in the ${ }^{1} \mathrm{H}-{ }^{15} \mathrm{~N}$ HSQC spectrum of Ap5abound $\mathrm{Y} 171 \mathrm{~W}$ reveal the presence of two structural states. Enlargements of the two spectral regions enclosed by dotted boxes are presented in subfigure b. (b) Exchange peaks in ZZ-exchange spectra of Ap5a-bound $\mathrm{Y} 171 \mathrm{~W}$ indicate the interconversion of the two structural states. Diagonal peaks are indicated by ' $a$ ' and ' $b$ ', whereas exchange cross-peaks are labelled as 'ab' and 'ba', respectively. (c) The rate constant for conformational exchange, $k_{\text {conf }}$ between the two structural states $\left(k_{\text {conf }}=25 \pm 10 \mathrm{~s}^{-1}\right)$ was determined by quantitative analysis of ZZ-exchange data for the displayed residues at $15^{\circ} \mathrm{C}$. note that the split peaks reflect two different Ap5a-bound states because the enzyme concentration used in the NMR experiments was $500 \mu \mathrm{M}$, which is far greater than the $K_{\mathrm{D}}$ value for the binding of Ap5a to Y171W $\left(K_{\mathrm{D}}=250 \pm 60 \mathrm{nM}\right)$. ZZ-exchange experiments $^{20,21}$ are designed to enable quantification of exchange kinetics in cases where two conformational states interchange slowly on the NMR timescale and thus give rise to two distinct peaks, as is the case for Y171W under Ap5asaturated conditions at $25^{\circ} \mathrm{C}$. In these experiments, exchange kinetics are quantified by monitoring the growth of exchange peaks and the simultaneous depletion of diagonal peaks as shown in Fig. 2b. When this experiment was performed on a sample consisting of Ap5a-bound Y171W, the two states (manifested as dual peaks) were found to be in dynamic equilibrium because they gave rise to exchange cross-peaks (Fig. 2b,c). The rate of conformational interconversion, $k_{\text {conf }}$, between the two states was determined to be $25 \pm 10 \mathrm{~s}^{-1}$ at $15^{\circ} \mathrm{C}$ (Fig. 2c) using the procedure of Miloushev et $a l^{22}$. The data were also analysed following the procedure of Farrow et al. ${ }^{20}$ (Supplementary Fig. $2 \mathrm{f}-\mathrm{j}$ ) and both methods resulted in very similar rates of interconversion. A second independent measure of the apparent rate constant, $k_{\text {app }}$, for Ap5a-induced conformational change of Y171W was obtained using fluorescence stopped-flow experiments, which yielded an Ap5a concentration-independent value of $k_{\text {app }}=24 \pm 3 \mathrm{~s}^{-1}$ at $17^{\circ} \mathrm{C}$ (Supplementary Fig. 2k). The initial Ap5a to Y171W binding event occurs within the dead time of the measurement and is therefore silent in the stopped-flow experiment (Supplementary Fig. 2l). Overall, it appears that the Y171W mutation shifts the dynamic response of AdK into a regime where fluctuations in the Ap5a-bound state become slow on the NMR timescale and hence directly observable.

Characterizing a high-energy state of Ap5a-bound Y171W. What are the underlying structural properties of the two interconverting structural states that give rise to split peaks? To characterize the two states observed in the NMR spectrum of Ap5a-bound Y171W, we exploited the fact that a protein's diffusion coefficient provides an accurate measure of its hydrodynamic dimension ${ }^{23}$. Diffusion experiments were performed on the apo- and Ap5a-bound forms of wild-type AdK to obtain reference data for the open and closed structural ground states, respectively; the more compact closed state was found to diffuse significantly faster than the open apo state (Fig. 3a). To assess the accuracy of these measurements, we also determined the diffusion coefficients of the P177A mutant, which were almost identical to those of the wild type (Fig. 3a). It is important to note that the ratio of the radii of gyration for the open and closed states is consistent with the ratios of the measured hydrodynamic radii for the wild type and AdK P177A (Supplementary Table 1). Therefore, diffusion measurements can be used to characterize the proteins' molecular topology. Strikingly, the diffusion coefficient of Ap5a-bound Y171W is intermediate between that of apo- and Ap5a-bound wild-type AdK (Fig. 3a), and can accurately be modelled as a linear combination of the values for the open and closed states of the wild type in which both have approximately equal weights. Since we observe two sets of resonances in the NMR spectrum of Ap5a-bound Y171W and its diffusion coefficient is equal to the average of those for the open and closed states of the wild type, we assigned the split peaks in its NMR spectrum to two distinct structural states-an Ap5a-bound open conformation and an Ap5a-bound closed conformation. This is the first time that closed and open substrate-bound states have been observed simultaneously. The relationship between the protein's hydrodynamic properties and its molecular structure is 

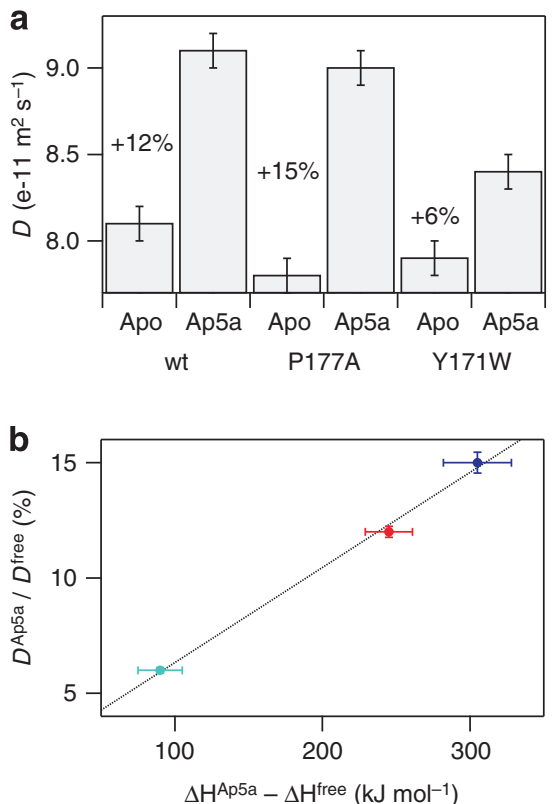

C

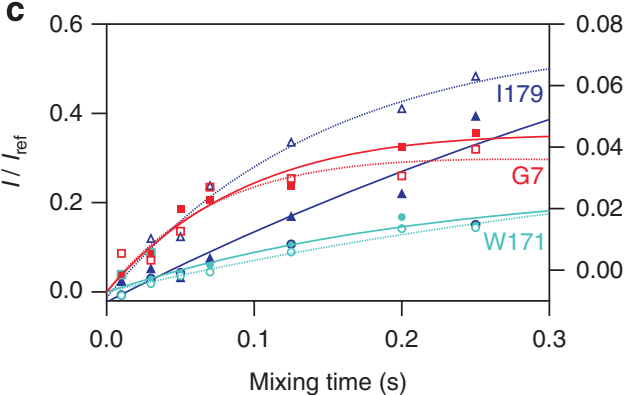

Figure 3 | Ap5a-bound Y171W simultaneously populates both open and closed conformations. (a) The diffusion coefficients of the wild type, P177A and Y171W AdK variants show that Ap5a-bound Y171W populates both open and closed structures. The bound open conformation corresponds to the high-energy state shown in red in Fig. 1c. (b) The relative change in diffusion coefficient between the apo- and Ap5a-bound states correlates with the difference in unfolding enthalpy between these states (wild type in red, P177A in blue and Y171W in cyan). (c) Build-up curves for residuespecific proton exchange on the millisecond timescale. Solid and dotted lines correspond to the two exchanging peaks observed for Ap5a-bound Y171W. Residue lle179 (labelling on right $y$ axis) is the only residue shown on the graph whose solvent exchange rate differs significantly between the two states.

underlined by the correlation between the change in the diffusion coefficient comparing apo and Ap5a-bound states and that in the enthalpy of unfolding (Fig. 3b). It was also expected that the solution properties of the Ap5a-bound open and closed states would not only differ with respect to diffusion, and millisecond solvent exchange rates determined using MEXICO $^{24}$ showed that the stabilities of residues close to the substrate-binding pocket (Leu83, Asp110, Val111 and Ile179) differ between the substratebound open and closed states (Fig. 3c, Supplementary Fig. 3 and Supplementary Table 2). These results support the assignment of the two sets of NMR peaks to the Ap5a-bound open and closed states because the solvent accessibility of the substrate-binding pocket is expected to increase when the enzyme samples the two conformations. The secondary structure and therefore also the backbone hydrogen bonding patterns of AdK is virtually identical in open and closed states. The amide protons of residues Leu83, Asp110 and Ile179 are hydrogen bonded in both open and closed states, whereas the amide proton of residue Val111 is not engaged in a hydrogen bond in neither of the two states. It thus appears that amide proton exchange is modulated by the combined action of Ap5a interaction and the structural interconversion between Ap5a-bound open and closed states.

The results presented above raise an important question: is the Ap5a-bound open structure observed for the Y171W mutant also populated in wild-type AdK? This was answered by examining the rate of interconversion between the structural states of Ap5a-bound Y171W at different temperatures. Raising the temperature from 10 to $50^{\circ} \mathrm{C}$ caused peaks that are split in the ${ }^{1} \mathrm{H}-{ }^{15} \mathrm{~N}$ HSQC spectra of Y171W at low temperatures to merge into single peaks, showing the transition in the exchange regime from slow (on the NMR timescale) to fast as the temperature increases (Supplementary Fig. 4). Both enzyme variants thus behave similarly at high temperature, and we can conclude that the chemical shifts observed under Ap5a-saturated conditions represent an average of the shifts for the open and closed Ap5a-bound states.

The performed line shape analysis provided rates of conformational exchange of 10 and $20 \mathrm{~s}^{-1}$ at 10 and $20^{\circ} \mathrm{C}$ (Supplementary Fig. 4), respectively, in good agreement with the ZZ-exchange experiment (Fig. $2 \mathrm{c}$ and Supplementary Fig. $2 \mathrm{f}-\mathrm{j}$ ).

$\boldsymbol{k}_{\text {cat }}$ versus $K_{\mathrm{M}}$ and $\boldsymbol{k}_{\text {cat }}$ versus $\boldsymbol{K}_{\mathrm{D}}$ compensation. Since AdK is rate limited by the rate constant for the opening of its substratebinding subdomains $\left(k_{\mathrm{open}}\right)$, its binding affinities (quantified in terms of $K_{\mathrm{M}}$ or $K_{\mathrm{D}}$ ) and $k_{\mathrm{cat}}$ values are both modulated in response to changes in $k_{\text {open }}$ (Fig. 1c). The reason is that $k_{\text {cat }}$ is equal to $k_{\text {open }}$ and that binding affinities depend on the statistical weight of the closed state that is governed by $k_{\text {open }}$ and $k_{\text {close. }}$ The dependency of $k_{\text {cat }}$ and $K_{\mathrm{M}}$ on the equilibrium constant between open and closed states has been reported previously. It was found that perturbations that affect the native free-energy landscape of AdK give rise to ' $k_{\text {cat }}$ versus $K_{\mathrm{M}}$ compensation' whereby $k_{\text {cat }}$ and $K_{\mathrm{M}}$ are anti-correlated such that the specificity constant $\left(k_{\mathrm{cat}} / K_{\mathrm{M}}\right)$ is silent to the perturbation ${ }^{13}$. Since a similarly strong correlation was observed between the $k_{\mathrm{cat}}$ and $K_{\mathrm{M}}$ values for the AdK variants examined in this work (Supplementary Fig. 5a), we conclude that the effect of the Y171W and P177A replacements merely perturbs the functional energy landscape of wild-type AdK, and that the conclusions drawn from experiments on Y171W are relevant to the functional cycle of wild-type AdK. Furthermore, the observation of $k_{\text {cat }}$ versus $K_{\mathrm{D}}$ compensation for Ap5a binding (Supplementary Fig. 5b) is important because it shows that the structure and properties of this substrate analogue adequately mimic those of the enzyme's natural substrates and products (AMP, ATP and ADP) during its functional catalytic cycle. Interestingly, we observed a hyperbolic temperature dependency in the $K_{\mathrm{D}}$ values for the wild type, P177A and Y171W variants, with the binding affinity peaking at the growth temperature of Escherichia coli (Supplementary Fig. 5c). It thus appears that the dynamic properties underlying the enzyme's binding affinity are optimized for functionality at $37^{\circ} \mathrm{C}$, which substantiates the inference that enzymes' dynamics develop under evolutionary constraints ${ }^{25}$.

\section{Discussion}

Protein engineering has enabled us to directly observe, characterize and manipulate a functionally indispensable highenergy state of AdK. This is a significant development because while such states are known to be essential for enzymatic catalysis ${ }^{4,6,9}$, it was previously impossible to study them directly. Our approach is based on specific amino-acid replacements in a region that is known to affect the allosteric opening and closing 
reaction of AdK. This confined spatial hotspot for manipulation of an enzyme's dynamics is analogous to that found in maltose binding protein, where the replacement of two specific residues markedly change the enzyme's ligand binding affinity and conformational dynamics ${ }^{26}$. The high-energy AdK state corresponds to an open substrate-bound conformation that is in equilibrium with a stable substrate-bound ground state populating a closed structure. With the experimental set-up, it is not possible to determine whether the domain motions are correlated, anti-correlated or uncorrelated. There must be a structural reason for the change in dynamics that is observed in the Y1711W variant and that enabled the direct detection of the high-energy state. In this particular case, the altered dynamics likely depend on the two different side-chain conformations of Y171W in the Ap5a-bound state. In the crystal structure of Ap5a-bound Y171W, the enzyme is fully closed, whereas NMR measurements show that Ap5a-bound Y171W exchanges between open and closed states in solution. Hence, it appears that one of the side-chain conformations observed in the crystal structure triggers opening of the enzyme in solution. We were able to determine the molecular topology of the high-energy state and also to directly quantify the kinetic parameters of the conformational dynamics connecting it to the stable ground state. A key prediction of the catalytic model developed for AdK, which suggests that conformational dynamics are rate limiting in the catalytic cycle, is that free-energy barriers determined by measuring the rate of conformational interconversion should converge with those determined by examining the enzyme's catalytic turnover. This prediction was confirmed: the barrier height estimated by analysing the temperature dependence of the enzyme's conformational dynamics (Fig. 4a,b; $63.8 \pm 0.2 \mathrm{~kJ} \mathrm{~mol}^{-1}$ ) is in good agreement with that determined on the basis of measured enzyme activity (Fig. 4c; $62.4 \pm 3.3 \mathrm{~kJ} \mathrm{~mol}^{-1}$ ). From a mechanistic standpoint, these results resolve the long-standing question about whether the induced $\mathrm{fit}^{27}$ or conformational selection ${ }^{28,29}$ models are most appropriate for describing the binding of substrates to enzymes. The hallmark of the conformational selection model is that active conformations are sampled even in the absence of the substrate, and that substrate addition merely redistributes the relative population of inactive versus active states. It has previously been suggested that substrate-free AdK samples closed-like structures. In a complete description of ligand binding to AdK through competing conformational selection or induced fit models ${ }^{30}$ it is assumed that the closed apo structure is a binding competent state. This has however not been shown experimentally and it is possible that the substrate-binding sites are occluded due to steric hindrance in the apo-closed structure ${ }^{31}$. Our kinetic stopped-flow data show that AdK is using an induced fit mechanism for closure following the initial binding event, thus the conformational change occurs after ligand binding. Hence, the conformational change occurs by two different mechanisms in apo- and substrate-bound states. In absence of substrate domain closure occurs by conformational selection, whereas induced fit is utilized in the presence of substrate. However, in a competition between induced fit and conformational selection pathways, the flux through either path is determined by intracellular substrate concentrations and all contributing rate constants as discussed in ref. 30. The rate constants for the enzyme's conformational dynamics appear to have been determined by natural selection because its substrate-binding affinity (which is governed by its conformational dynamics) is highest at the preferred growth temperature of $E$. coli. The direct NMR-based characterization of both the structure and dynamics of a high-energy state that is functionally relevant to AdK catalysis enables us to establish a detailed reaction scheme based on the conformational
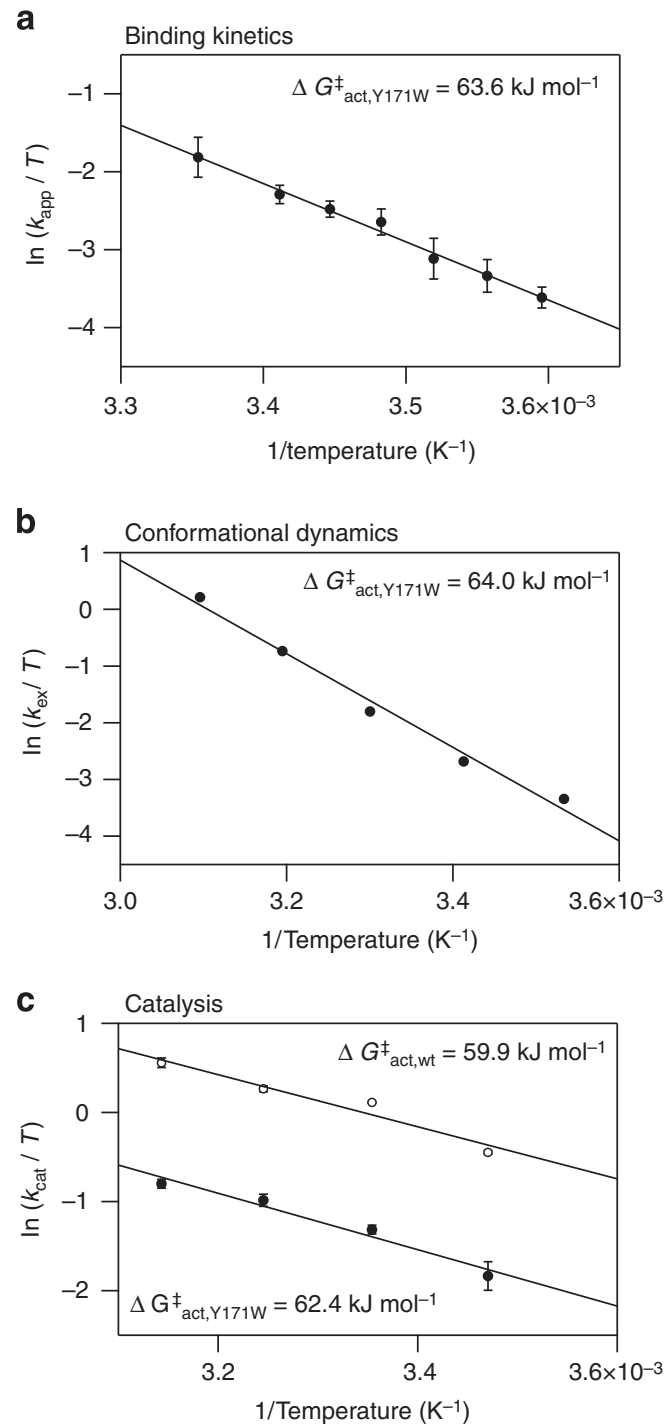

Figure 4 | Activation barriers for conformational dynamics and catalysis. Barrier heights were determined at $25^{\circ} \mathrm{C}$ from Eyring-Polanyi analyses by monitoring (a) apparent rate constants for Ap5a binding to Y171W based on fluorescence stopped-flow experiments, which yielded kinetic parameters of $\Delta H^{\mathrm{sf}}=(62.1 \pm 2.8) \mathrm{kJ} \mathrm{mol}^{-1}$ and $\Delta S^{\mathrm{sf}}=-(4.7 \pm 0.2) \mathrm{J} \mathrm{mol}^{-1} \mathrm{~K}^{-1}$; and (b) conformational exchange dynamics for residue Thr89 of Y171W based on NMR line shape analysis. Eyring-Polanyi analysis in this case yields kinetic parameters of $\Delta H^{\mathrm{LS}}=(68.6 \pm 4.7) \mathrm{kJ} \mathrm{mol}^{-1}$ and $\Delta S^{\mathrm{LS}}=(15.3 \pm 1.1) \mathrm{J} \mathrm{mol} \mathrm{Ju}^{-1}$ $\mathrm{K}^{-1}$. (c) Catalytic turnover values for the wild type (open circles) and $\mathrm{Y} 171 \mathrm{~W}$ (closed circles) AdK variants determined using a coupled assay ${ }^{35}$.

Eyring-Polanyi analysis yields kinetic parameters of $\Delta H^{\text {act }}{ }_{w t}=(24.1 \pm 4.2)$ $\mathrm{kJ} \mathrm{mol}^{-1}$ and $\Delta S^{\text {act }}=-(120 \pm 20) \mathrm{J} \mathrm{mol}^{-1} \mathrm{~K}^{-1}$ for wild type, and $\Delta H^{\text {act }}{ }_{Y 171 \mathrm{~W}}=(26.6 \pm 3.3) \mathrm{kJ} \mathrm{mol}^{-1}$ and $\Delta S_{\mathrm{Yact}}^{\text {act }}{ }_{\mathrm{H} 1 \mathrm{~W}}=-(120 \pm 20) \mathrm{J} \mathrm{mol}^{-1}$ $\mathrm{K}^{-1}$ for $\mathrm{Y} 171 \mathrm{~W}$ variant.

states that constitute the structural framework for AdK catalysis (Fig. 5).

The $k_{\text {cat }}$ versus $K_{\mathrm{D}}$ compensation discovered here is adding additional insights compared to the $k_{\text {cat }}$ versus $K_{\mathrm{M}}$ compensation discovered previously for $\mathrm{AdK}^{13}$. The compensatory effect on activity and binding affinities is phenomenologically related to results reported for the Dbl homology domain of the guanine exchange factor Vav1 where it was found that the specificity constant $\left(k_{\mathrm{cat}} / K_{\mathrm{M}}\right)$ depends linearly on the population of a highenergy state ${ }^{32}$. A related dependency on the specificity constant on the population of a high-energy state has also been reported 


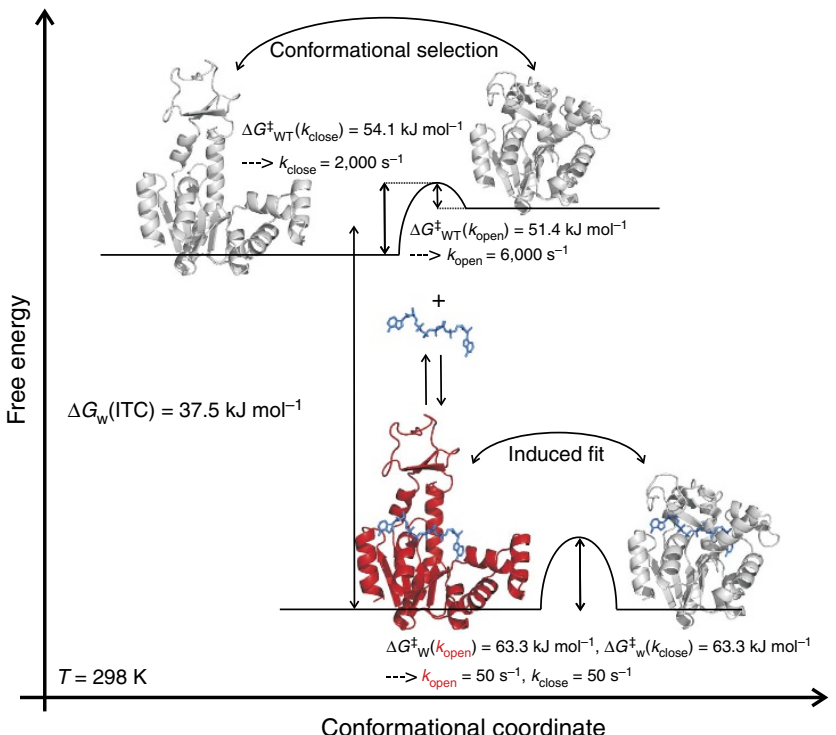

Figure 5 | The high-energy state is crucial for the catalytic mechanism of AdK. The state shown in red corresponds to the directly observed highenergy state of Y171W and is modelled from known structures (1AKE and $4 A K E$ ). This state completes the description of conformations required for AdK catalysis. Relative ground state free energies and activation barriers, $\Delta G^{\ddagger}$, are indicated for apo- ${ }^{11}$ and substrate-bound AdK. The binding of Ap5a to $\mathrm{Y} 171 \mathrm{~W}$ lowers the free energy of the system by $37.5 \mathrm{~kJ} \mathrm{~mol}^{-1}$. Kinetic rate constants are displayed for the dynamic interconversion between structural states. Taken together, the data show that population of closed AdK conformations is accomplished through conformational selection ${ }^{29}$ in the apo state and induced fit in the presence of substrate ${ }^{27}$. Subscripts 'WT' and ' $W$ ' correspond to wild type and Y171W, respectively. Although no direct high-resolution structural data exist for the closed apo structure, indirect evidence from ensemble ${ }^{49}$ and single-molecule ${ }^{11}$ fluorescence resonance energy transfer experiments and chemical shifts ${ }^{13}$ points towards that compact 'closed-like' structures are sampled by AdK in the absence of substrate.

for phosphorylation of phospholamban by cAMP-dependent protein kinase $\mathrm{A}^{33}$. As a final note, we believe that our results indicate that there is great potential for exploiting the rational tuning of conformational dynamics in the de novo design of enzymes.

\section{Methods}

Sample preparation. Wild-type AdK, P177A and Y171W were overexpressed in BL21(DE3) cells from plasmids controlled by the AdK promotor. Cells were harvested by centrifugation and resuspended in a buffer consisting of $50 \mathrm{mM}$ Tris, $\mathrm{pH}$ 7.5. After a freeze-thaw cycle, the cells were lysed by sonication and centrifuged to remove cell debris. Purification of supernatants from sonication was accomplished with Blue Sepharose using a linear $\mathrm{NaCl}$ gradient for elution. Pooled fractions containing AdK were subjected to size-exclusion chromatography in a

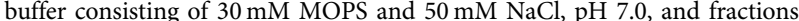
containing AdK were pooled, concentrated and stored at $-20^{\circ} \mathrm{C}$. All protein concentrations of wild type and P177A were determined using an extinction coefficient of $10,430 \mathrm{M}^{-1} \mathrm{~cm}^{-1}$ and for Y171W of $14,440 \mathrm{M}^{-1} \mathrm{~cm}^{-1}$ at $\lambda=280 \mathrm{~nm}$. Nucleotide concentrations were determined using an extinction coefficient of $30,000 \mathrm{M}^{-1} \mathrm{~cm}^{-1}$ (Ap5a) and $15,400 \mathrm{M}^{-1} \mathrm{~cm}^{-1}$ (ADP) at $\lambda=260 \mathrm{~nm}$.

Site-specific mutagenesis. Primer sequences for the P177A and Y171W mutants were ordered from Eurofins MWG Operon. The mutants were made using the QuikChange method (Stratagene), expressed in a pEAK-91 vector ${ }^{34}$ and verified by DNA sequencing (Eurofins MWG Operon).

Fluorescence kinetic stopped flow. A sequential mixing stopped-flow spectrometer (Chirascan, Applied Photophysics) was used for all kinetic experiments. The reaction kinetics of Ap5a binding to Y171W was observed by the change in tryptophan fluorescence intensity using a cutoff filter of $340 \mathrm{~nm}$ after excitation at a wavelength of $295 \mathrm{~nm}$. All experiments were carried out in $30 \mathrm{mM}$ MOPS and $50 \mathrm{mM} \mathrm{NaCl}, \mathrm{pH}$ 7.0. Mixing experiments were performed at 5, 8, 11, 14, 17, 20 and $25^{\circ} \mathrm{C}$. The temperature of the whole mixing device was maintained with a circulating water bath. Binding reactions were initiated by a rapid 1:10 mixing ratio of different concentrations of Ap5a with a solution containing $15 \mu \mathrm{M}$ Y $171 \mathrm{~W}$. An additional control mixing experiment was performed to exclude any effect of Ap5a on the buffer. No influence to fluorescence emission was observed. Kinetic time traces were collected at least 10 times under identical conditions and were averaged for the fitting procedure according to equation (1):

$$
I=I_{0} \exp \left(-k_{\text {app }} t\right),
$$

where $I$ is the fluorescence emission intensity and $k_{\text {app }}$ is the apparent rate constant for the binding event. Temperature-dependent apparent rate constants were plotted against the inverse absolute temperature and fitted using the linear form of the Eyring-Polanyi equation (2):

$$
\ln \left(k_{\mathrm{app}} / T\right)=-\Delta H^{\mathrm{sf}} /(R T)+\ln \left(k_{\mathrm{B}} / h\right)+\Delta S^{\mathrm{sf}} / R,
$$

with the absolute temperature $T$, Boltzmann constant $k_{\mathrm{B}}$, Planck constant $h$, the change in enthalpy $\Delta H^{\text {sf }}$ and the change in entropy $\Delta S^{\text {sf }}$ caused by Ap5a interaction to Y171W. The abbreviation 'sf' stands for stopped flow. Using the Gibbs-Helmholtz equation (3) leads to the determination of the activation barrier, $\Delta \mathrm{G}_{\text {act }}^{\ddagger}$, at a specific temperature $T$ :

$$
\Delta \mathrm{G}_{\text {act }}^{\ddagger}(T)=\Delta H-T \Delta S .
$$

Kinetic assay probing enzyme activity. Enzyme kinetic parameters for ATP turnover in the direction of ADP formation were quantified for wild-type AdK and $\mathrm{Y} 171 \mathrm{~W}$ at $15,25,35$ and $45^{\circ} \mathrm{C}$ and for P177A at $25^{\circ} \mathrm{C}$ using a coupled spectroscopic assay ${ }^{35}$ on a Varian Cary 50 UV-Visible Spectrophotometer. Practically, the ATP concentration was varied and ATP was added together with $10 \mu \mathrm{l}$ of protein to a solution containing $100 \mathrm{mM}$ Tris, $80 \mathrm{mM} \mathrm{KCl,} 0.2 \mathrm{mM} \mathrm{NADH}, 0.4 \mathrm{mM}$ PEP, $0.3 \mathrm{mM}$ AMP and $2 \mathrm{mM} \mathrm{MgCl}_{2}$ at $\mathrm{pH} 7.5$ giving a final volume of $471 \mu \mathrm{l}$. Monitoring the time-dependent change of the absorption value at a wavelength of $340 \mathrm{~nm}$ leads finally to the velocity of the reaction. This reaction velocity, $v$, was plotted against ATP concentration, $c_{\mathrm{ATP}}$, and using Michaelis-Menten's relation (equation (4)) enables extraction of maximal turnover rate, $k_{\text {cat }}$, and ATP affinity, $K_{\mathrm{M}}$, for the used spectroscopic assay:

$$
v=\left(k_{\text {cat }} \times c_{\text {ATP }}\right) /\left(K_{\mathrm{M}}+c_{\mathrm{ATP}}\right) .
$$

Eyring-Polanyi analysis was performed as described for fluorescence kinetic stopped flow.

Structure determination by X-ray crystallography. Crystallization. Before crystallization, the proteins were stored in buffer containing $50 \mathrm{mM} \mathrm{NaCl}$ and $30 \mathrm{mM}$ MOPS adjusted to $\mathrm{pH}$ 7.0. The protein concentrations of Y171W and P177A variants were adjusted to 12 and $22 \mathrm{mg} \mathrm{ml}^{-1}$, respectively. The concentrations were maintained in the presence of the inhibitor P1,P5-di(adenosine-5')pentaphosphate, Ap5a. Sitting drop crystallization set-ups in 96-well MRC two-well crystallization plates (Molecular Dimensions) were carried out with a Mosquito Nanodrop pipetting robot (TTP Labtech). Initial crystallization conditions were obtained from Crystal Screen and Crystal Screen 2 (Hampton Research) and manually optimized in 24-well Linbro plates (Hampton Research) using the hanging-drop vapour diffusion method. The final drops contained $2 \mu \mathrm{l}$ of Y171W or Y171W/Ap5a solution (five-time stoichiometric excess) mixed with $1 \mu \mathrm{l}$ of reservoir solution and equilibrated over $1 \mathrm{ml}$ reservoir buffer. Owing to its higher initial protein concentration, only $1 \mu \mathrm{l}$ of the P177A or P177A/Ap5a solution (five-time stoichiometric excess) was mixed with $1 \mu \mathrm{l}$ of precipitation buffer. The crystals diffracting best were obtained at $18^{\circ} \mathrm{C}$ and with the following solutions: Y171W in $0.1 \mathrm{M}$ Tris (pH 8.0), $0.2 \mathrm{M} \mathrm{NaOAc}$ and $28 \%$ PEG $4 \mathrm{~K}$; Y171W/Ap5a in $0.1 \mathrm{M}$ sodium citrate ( $\mathrm{pH}$ 6.0), $0.2 \mathrm{M}$ ammonium acetate and $28 \%$ PEG $4 \mathrm{~K}$; P177A in $0.1 \mathrm{M}$ Tris (pH 8.5), $0.2 \mathrm{M} \mathrm{MgCl}_{2}$ and $30 \%$ PEG $4 \mathrm{~K}$; and P177A/Ap5a in 0.1 M Tris (pH 8.5), 0.2 M ammonium acetate and 30\% PEG $4 \mathrm{~K}$.

Data collection, structure determination and refinement. Crystals were equilibrated in cryo-protectant buffer adjusted to $20 \%(\mathrm{v} / \mathrm{v})$ glycerol in the respective reservoir solution before flash-cooling to $100 \mathrm{~K}$. All X-ray diffraction data sets were collected at a wavelength of $0.15418 \mathrm{~nm}(\mathrm{Cu}-\mathrm{K} \alpha$ radiation) on an inhouse X8-Proteum system equipped with a MicroStar-H X-ray generator (Bruker AXS). Diffraction data were integrated with SAINT and scaled using SADAPS (Bruker AXS programme suite). Data collection statistics are listed in Table 1.

All four AdK structures were determined by molecular replacement with the programme PHASER from the PHENIX programme suite ${ }^{36}$ using the E. coli ApK apo and holo structures, pdb code 4AKE (ref. 37) and 1AKE (ref. 38) as search models. The electron density maps were well defined and allowed the atomic models to be built using the programme $\operatorname{Coot}^{39}$ and refined with PHENIX Refine $^{36}$. The AdK structures were superimposed and analysed with ICM-Pro (Molsoft LLC), which was also used to prepare the structural figures. The atomic coordinates and the structure factors have been deposited within the Protein Data Bank $^{40}$ (PDB codes 4X8M for Y171W-apo, 4X8O for Y171W-holo, 4X8H for P177A-apo and 4X8L for P177A-holo). 
CD spectroscopy. Protein unfolding of the apo and Ap5a-bound wild type, P177A and Y171W AdK proteins was monitored by measuring the ellipticity at $220 \mathrm{~nm}$ using a 1-mm path length quartz cuvette on a Jasco J-810 spectropolarimeter. The proteins were stored in $30 \mathrm{mM}$ MOPS and $50 \mathrm{mM} \mathrm{NaCl}, \mathrm{pH} 7.0$, and concentrations for the $\mathrm{CD}$ experiments ranged between 5 and $10 \mu \mathrm{M}$. Ap5a was titrated to a five-time stoichiometric excess. Equation (5) was used to get the midpoint, $T_{\mathrm{m}}$, and the van't Hoff enthalpy of unfolding, $\Delta H_{\mathrm{vH}}$, at $T_{\mathrm{m}}$.

$$
\begin{aligned}
& \Xi^{\mathrm{OBS}}(T)=\left(\Xi_{\mathrm{f}}+m_{\mathrm{f}} T+\left(\Xi_{\mathrm{u}}+m_{\mathrm{u}} T\right) K_{\mathrm{u}}\right) /\left(1+K_{\mathrm{u}}\right), \\
& \text { with : } K_{\mathrm{u}}=\exp \left(\left(\Delta H_{\mathrm{vH}}\left(1-T / T_{\mathrm{m}}\right)\right) /(R T)\right),
\end{aligned}
$$

where $\Xi^{\mathrm{OBS}}$ is the temperature-dependent observed ellipticity at $220 \mathrm{~nm}, m_{\mathrm{f}}, m_{\mathrm{u}}, \Xi_{\mathrm{f}}$ and $\Xi_{\mathrm{u}}$ are the slopes and intercepts of the baselines related to folded and unfolded conditions, $K_{\mathrm{u}}$ is the equilibrium constant in direction of unfolding, $T$ is the absolute temperature and $R$ represents the gas constant.

NMR spectroscopy. All NMR experiments were conducted at a Bruker Avance III HD $850 \mathrm{MHz}$ spectrometer equipped with a z-gradient cryogenic probe using $30 \mathrm{mM}$ MOPS and $50 \mathrm{mM} \mathrm{NaCl}, \mathrm{pH} 7.0$, and $5 \% \mathrm{D}_{2} \mathrm{O}(\mathrm{v} / \mathrm{v})$ as sample buffer. All two-dimensional (2D) and 3D NMR data sets were processed with NMRPipe ${ }^{41}$ and analysed in NMRView ${ }^{42}$.

Assignment experiments. Backbone ${ }^{15} \mathrm{~N},{ }^{1} \mathrm{H}$ chemical shifts have been previously published for wild-type AdK for both apo- ${ }^{43}$ and Ap5a-bound conditions ${ }^{44}$, and were verified using ${ }^{1} \mathrm{H}_{-}{ }^{15} \mathrm{~N}$ NOESY-HSQC spectroscopy. Of 204 non-prolyl residues, 198 (apo) and 195 (Ap5a bound) could be assigned. Backbone ${ }^{15} \mathrm{~N},{ }^{1} \mathrm{H}$ chemical shifts for P177A and Y171W in the apo- and Ap5a-bound states were obtained by comparison with the wild-type chemical shifts and acquisition of ${ }^{1} \mathrm{H}_{-}{ }^{15} \mathrm{~N}$ NOESY-HSQC spectra. For P177A, 198 (apo) and 181 (Ap5a bound) could be assigned out of 205 non-prolyl residues. For Y171W, 200/204 (apo) and 265 (Ap5a bound) out of 204 non-prolyl residues could be assigned.

The assigned chemical shifts have been deposited within the BMRB database for P177A-apo (accession number 25353), Y171W-apo (accession number 25357), P177A-holo (accession number 25360), Y171W-holo data set a (accession number 25361) and Y171W-holo data set b (accession number 25362).

Weighted differences in chemical shifts $\Delta \omega$ is calculated by using equation (6)

$$
\Delta \omega=\left(\left(\left(\Delta^{1} \mathrm{H}\right)^{2}+\left(\Delta^{15} \mathrm{~N} / 5\right)^{2}\right) / 2\right)^{0.5},
$$

where $\Delta^{1} \mathrm{H}$ is the change in proton and $\Delta^{15} \mathrm{~N}$ is the change in nitrogen dimension, respectively.

Diffusion experiments. NMR diffusion spectra were recorded using pulsed field bipolar gradient stimulated echo experiments ${ }^{45}$ at $25^{\circ} \mathrm{C}$. For each diffusion profile, 21 different gradient strengths $G$ were used for $6 \mathrm{~ms}$ along the $z$ axis followed by a $100 \mu$ s recovery delay. The diffusion of AdK molecules was allowed to proceed for $100 \mathrm{~ms}$ to ensure a protein signal intensity decay of $\sim 90 \%$ at maximum gradient strength. The calibration of $G$ was performed by a standard protocol ${ }^{46}$. For error estimation, four different gradient strengths were repeated (relative gradient strengths of $1,10,40$ and $70 \%$ ). The measured ${ }^{1} \mathrm{H}$ NMR spectra were integrated within the aliphatic signal region ( $-0.5 \ldots 2.5$ p.p.m.) and fitted to equation (7):

$$
I(G)=I(0) \exp \left(-G^{2} \gamma^{2} \delta^{2} D(\Delta-\delta / 3)\right),
$$

where $\gamma$ is the gyromagnetic ratio, $\delta$ is the gradient length, $\Delta$ is the diffusion time and $D$ is the calculated diffusion coefficient ${ }^{45}$. Water suppression was achieved by presaturation and WATERGATE. Spectra were processed and analysed by Topspin 3.2.

Line shape analysis. For line shape analysis, ${ }^{15} \mathrm{~N}$ slices were extracted from $2 \mathrm{D}$ ${ }^{1} \mathrm{H}-{ }^{15} \mathrm{~N}$ HSQC spectra recorded at $10,20,30,40$ and $50^{\circ} \mathrm{C}$ for Ap5a-bound Y171W. Subsequently, a two-component Lorentzian function was fitted to individual $1 \mathrm{D}{ }^{15} \mathrm{~N}$ spectra to obtain intensity offsets, $y_{1}$ and $y_{2}$, chemical shift offsets, $\delta_{1}$ and $\delta_{2}$, peak amplitudes, $A_{1}$ and $A_{2}$ and line widths, $w_{1}$ and $w_{2}$ :

$$
y=y_{1}+A_{1} /\left(\left(\delta-\delta_{1}\right)^{2}+w_{1}\right)+y_{1}+A_{1} /\left(\left(\delta-\delta_{1}\right)^{2}+w_{1}\right) .
$$

The rate of conformational exchange, $k_{\mathrm{ex}}$, between the states 1 and 2 was introduced by the McConnell equations ${ }^{47}$. Populations and chemical shift offsets for both states were used from Lorentzian regression as discussed above during simulation of ${ }^{15} \mathrm{~N}$ NMR line shape.

Eyring-Polanyi analysis was performed as described for fluorescence kinetic stopped flow.

ZZ-exchange spectroscopy. ZZ-exchange experiments for Ap5a-bound Y171W were set-up according to Farrow et al. ${ }^{20}$ and run at $15^{\circ} \mathrm{C}$ with mixing times, $t_{\text {mix }}$, of $20,30,40$ and $100 \mathrm{~ms}$. The rate constant of conformational interconversion, $k_{\text {conf }}$, was calculated using the procedure of Miloushev et $a l^{22}$ :

$$
I_{\mathrm{AB}}\left(t_{\text {mix }}\right) I_{\mathrm{BA}}\left(t_{\text {mix }}\right) /\left(I_{\mathrm{AA}}\left(t_{\text {mix }}\right) I_{\mathrm{BB}}\left(t_{\text {mix }}\right)-I_{\mathrm{AB}}\left(t_{\text {mix }}\right) I_{\mathrm{BA}}\left(t_{\text {mix }}\right)\right)=k_{\text {conf }}^{2} t_{\text {mix }}^{2},
$$

where $I_{\mathrm{AA}}, I_{\mathrm{BB}}$ are auto peak and $I_{\mathrm{AB}}, I_{\mathrm{BA}}$ are cross-peak intensities for individual residues.

MEXICO. Fast millisecond proton exchange was determined from $2 \mathrm{D}{ }^{1} \mathrm{H}-{ }^{15} \mathrm{~N}$ HSQC spectra using a modified MEXICO ${ }^{24}$ pulse sequence applying $10,30,50,70$, 125,200 and $250 \mathrm{~ms}$ mixing time delays. Cross-peak intensities were referenced to a conventional $2 \mathrm{D}^{1} \mathrm{H}_{-}{ }^{15} \mathrm{~N}$ HSQC and analysed according to Hofmann et al. ${ }^{48}$.
Isothermal titration calorimetry. Isothermal titration calorimetry experiments were carried out at 15, 25, 35 and $45^{\circ} \mathrm{C}$ using MicroCal Auto-iTC200 isothermal titration calorimeter (GE Healthcare). The reference cell was filled with sample buffer ( $30 \mathrm{mM}$ MOPS, $50 \mathrm{mM} \mathrm{NaCl}$, pH 7.0). Protein solutions $(380 \mu \mathrm{l})$ were filled into the sample cell and titrated with Ap5a loaded in the syringe $(100 \mu \mathrm{l})$.

The following set-up was used: reference power of $4 \mu \mathrm{cal} \mathrm{s} \mathrm{s}^{-1}$, initial delay of $200 \mathrm{~s}$, stirring speed of 400 r.p.m., spacing of $150 \mathrm{~s}$, filter of $5 \mathrm{~s}$, injection of $0.3 \mu \mathrm{l}$ (first injection) or $1.5 \mu \mathrm{l}$ (following injections) and low-feedback mode.

Particular protein/Ap5a concentration ratios were $60 \mu \mathrm{M} / 541 \mu \mathrm{M}, 90 \mu \mathrm{M} /$ $541 \mu \mathrm{M}$ and $120 \mu \mathrm{M} / 1,083 \mu \mathrm{M}$ for Y171W, $75 \mu \mathrm{M} / 541 \mu \mathrm{M}$ for wild type and $97 \mu \mathrm{M} / 541 \mu \mathrm{M}$ for P177A titration at $25^{\circ} \mathrm{C} ; 65 \mu \mathrm{M} / 1,109 \mu \mathrm{M}$ for all performed experiments at $15^{\circ} \mathrm{C}$ and $35^{\circ} \mathrm{C} ; 65 \mu \mathrm{M} / 650 \mu \mathrm{M}$ for Y171W and wild type; and $45 \mu \mathrm{M} / 555 \mu \mathrm{M}$ for P177A at $45^{\circ} \mathrm{C}$. Control experiments (injection of Ap5a into buffer solution) did not show any change in enthalpy. Raw data were collected, corrected for ligand heats of dilution and integrated using the MicroCal Origin software. Data sets were fitted by using a single-site binding model to yield the binding constant, $K_{\mathrm{D}}$, the apparent enthalpy of binding, $\Delta H$, and the stoichiometry of binding, $n$.

\section{References}

1. Luz, Z. \& Meiboom, S. Nuclear magnetic resonance study of the protolysis of trimethylammonium ion in aqueous solution-order of the reaction with respect to solvent. J. Chem. Phys. 39, 366-370 (1963).

2. Loria, J. P., Rance, M. \& Palmer, A. G. I. A relaxation-compensated CarrPurcell-Meiboom-Gill sequence for characterizing chemical exchange by NMR spectroscopy. J. Am. Chem. Soc. 121, 2331-2332 (1999).

3. Eisenmesser, E. Z., Bosco, D. A., Akke, M. \& Kern, D. Enzyme dynamics during catalysis. Science 295, 1520-1523 (2002).

4. Eisenmesser, E. Z. et al. Intrinsic dynamics of an enzyme underlies catalysis. Nature 438, 117-121 (2005).

5. Wolf-Watz, M. et al. Linkage between dynamics and catalysis in a thermophilic-mesophilic enzyme pair. Nat. Struct. Mol. Biol. 11, 945-949 (2004).

6. Boehr, D. D., McElheny, D., Dyson, J. H. \& Wright, P. E. The dynamic energy landscape of dihydrofolate reductase catalysis. Science 313, 1638-1642 (2006)

7. Watt, E. D., Shimada, H., Kovrigin, E. L. \& Loria, J. P. The mechanism of rate-limiting motions in enzyme function. Proc. Natl. Acad. Sci. USA 104, 11981-11986 (2007).

8. Korzhnev, D. M. et al. Low-populated folding intermediates of Fyn SH3 characterized by relaxation dispersion NMR. Nature 430, 586-590 (2004).

9. Beach, H., Cole, R., Gill, M. L. \& Loria, J. P. Conservation of $\mu$ s - ms enzyme motions in the apo- and substrate-mimicked state. J. Am. Chem. Soc. 127, 9167-9176 (2005).

10. Noda, L. H. Adenylate Kinase 279-305 (Academic Press, 1973)

11. Henzler-Wildman, K. et al. Intrinsic motions along an enzymatic reaction trajectory. Nature 450, 838-844 (2007).

12. Esteban-Martin, S. et al. Correlated inter-domain motions in adenylate kinase. PLoS Comput. Biol. 10, e1003721 (2014).

13. Ådén, J., Verma, A., Schug, A. \& Wolf-Watz, M. Modulation of a pre-existing conformational equilibrium tunes adenylate kinase activity. J. Am. Chem. Soc. 134, 16562-16570 (2012)

14. Hanson, J. A. et al. Illuminating the mechanistic roles of enzyme conformational dynamics. Proc. Natl. Acad. Sci. USA 104, 18055-18060 (2007).

15. Bhabha, G. et al. A dynamic knockout reveals that conformational fluctuations influence the chemical step of enzyme catalysis. Science 332, 234-238 (2011).

16. Brokaw, J. B. \& Chu, J.-W. On the roles of substrate binding and hinge unfolding in conformational changes of adenylate kinase. Biophys. J. 99, 3420-3429 (2010).

17. Schrank, T. P., Bolen, W. D. \& Hilser, V. J. Rational modulation of conformational fluctuations in adenylate kinase reveals a local unfolding mechanism for allostery and functional adaptation in proteins. Proc. Natl. Acad. Sci. USA 106, 16984-16989 (2009).

18. Sanders, C. R. I., Tian, G. \& Tsai, M.-D. Mechanism of adenylate kinase. Is there a relationship between local substrate dynamics, local binding energy, and the catalytic mechanism? Biochemistry 28, 9028-9043 (1989).

19. Lienhard, G. E. \& Secemski, I. I. P1,P5-Di(adenosine-5')pentaphosphate, a potent multisubstrate inhibitor of adenylate kinase. J. Biol. Chem. 248, 1121-1123 (1973).

20. Farrow, N. A., Zhang, O., Forman-Kay, J. D. \& Kay, L. E. A heteronuclear correlation experiment for simultaneous determination of $15 \mathrm{~N}$ longitudinal decay and chemical exchange rates of systems in slow equilibrium. J. Biomol. NMR 4, 727-734 (1994).

21. Montelione, G. T. \& Wagner, G. 2D chemical exchange NMR spectroscopy by proton-detected heteronuclear correlation. J. Am. Chem. Soc. 111, 3096-3098 (1989).

22. Miloushev, V. Z. et al. Dynamic properties of a type ii cadherin adhesive domain: implications for the mechanism of strand-swapping of classical cadherins. Structure 16, 1195-1205 (2008). 
23. Wilkins, D. K. et al. Hydrodynamic radii of native and denatured proteins measured by pulse field gradient $\mathrm{nmr}$ techniques. Biochemistry $\mathbf{3 8}$, 16424-16431 (1999).

24. Gemmecker, G., Jahnke, W. \& Kessler, H. Measurement of fast proton exchange rates in isotopically labeled compounds. J. Am. Chem. Soc. 115, 11620-11621 (1993).

25. Bhabha, G. et al. Divergent evolution of protein conformational dynamics in dihydrofolate reductase. Nat. Struct. Mol. Biol. 20, 1243-1249 (2013).

26. Seo, M.-H., Park, J., Kim, E., Hohng, S. \& Kim, H.-S. Protein conformational dynamics dictate the binding affinity for a ligand. Nat. Commun. 5, 3724-3730 (2014).

27. Koshland, D. E. Enzyme flexibility and enzyme action. J. Cell. Physiol. 54, 245-258 (1959).

28. Lange, O. F. et al. Recognition dynamics up to microseconds revealed from an RDC-derived ubiquitin ensemble in solution. Science 320, 1471-1475 (2008).

29. Monod, J., Wyman, J. \& Changeux, J.-P. On the Nature of allosteric transitions: a plausible model. J. Mol. Biol. 12, 88-118 (1965).

30. Hammes, G. G., Chang, Y.-C. \& Oas, T. G. Conformational selection or induced fit: a flux description of reaction mechanism. Proc. Natl. Acad. Sci. USA 106, 13737-13741 (2009).

31. Sullivan, S. M. \& Holyoak, T. Enzymes with lid-gated active sites must operate by an induced fit mechanism instead of conformational selection. Proc. Natl. Acad. Sci. USA 105, 13829-13834 (2008).

32. Li, P., Martins, I. R. S., Amarashinge, G. K. \& Rosen, M. K. Internal dynamics control activation and activity of the autoinhibited Vav DH domain. Nat. Struct. Mol. Biol. 15, 613-618 (2008).

33. Masterson, L. R. et al. cAMP-dependent protein kinase a selects the excited state of the membrane substrate phospholamban. J. Mol. Biol. 412, 155-164 (2011).

34. Reinstein, J., Brune, M. \& Wittinghofer, A. Mutations in the nucleotide binding loop of adenylate kinase of Escherichia coli. Biochemistry 27, 4712-4720 (1988).

35. Rhoads, D. G. \& Lowenstein, J. M. Initial velocity and equilibrium kinetics of myokinase. J. Biol. Chem. 243, 3963-3972 (1968).

36. Adams, P. D. et al. PHENIX: a comprehensive Python-based system for macromolecular structure solution. Acta Crystallogr. D Biol. Crystallogr. 66, 213-221 (2010)

37. Müller, C. W., Schlauderer, G. J., Reinstein, J. \& Schulz, G. E. Adenylate kinase motions during catalysis: an energetic counterweight balancing substrate binding. Structure 4, 147-156 (1996).

38. Müller, C. W. \& Schulz, G. E. Structure of the complex between adenylate kinase from Escherichia coli and the inhibitor Ap5A refined at $1.9 \AA$ resolution: A model for a catalytic transition state. J. Mol. Biol. 224, 159-177 (1992).

39. Emsley, P., Lohkamp, B., Scott, W. G. \& Cowtan, K. Features and development of Coot. Acta Crystallogr. D Biol. Crystallogr. 66, 486-501 (2010).

40. Berman, H. M. et al. The protein data bank. Nucleic Acids Res. 28, 235-242 (2000).

41. Delaglio, F. et al. NMRPipe: a multidimensional spectral processing system based on UNIX pipes. J. Biomol. NMR 6, 277-293 (1995).

42. Johnson, B. A. NMRView: a computer program for the visualization and analysis of NMR data. J. Biomol. NMR 4, 603-614 (1994).

43. Burlacu-Miron, S. et al. $1 \mathrm{H}, 13 \mathrm{C}$ and $15 \mathrm{~N}$ backbone resonance assignment of Escherichia coli adenylate kinase, a $23.6 \mathrm{kDa}$ protein. J. Biomol. NMR 13, 93-94 (1998).

44. Meirovitch, E., Sinev, M. A. \& Sineva, E. V. Sequence-specific 1H, 15N and 13C assignment of adenylate kinase from Escherichia coli in complex with the inhibitor AP5A. J. Biomol. NMR 13, 195-196 (1998).
45. Jones, J. A., Wilkins, D. K., Smith, L. J. \& Dobson, C. M. Characterisation of protein unfolding by NMR diffusion measurements. J. Biomol. NMR 10, 199-203 (1997).

46. Berger, S. \& Braun, S. 200 and More NMR Experiments. A Practical Course (Wiley-VCH, 2004).

47. McConnel, H. M. Reaction rates by nuclear magnetic resonance. J. Chem. Phys. 28, 430-431 (1958).

48. Hofmann, H. et al. Fast amide proton exchange reveals close relation between native-state dynamics and unfolding kinetics. J. Am. Chem. Soc. 131, 140-146 (2008).

49. Nagarajan, S. et al. Modulation of functionally significant conformational equilibria in adenylate kinase by high concentrations of trimethylamine oxide attributed to volume exclusion. Biophys. J. 100, 2991-2999 (2011).

\section{Acknowledgements}

This work was financially supported by the Swedish Research Council (M.W.-W. and A.E.S.-E.) and the Deutsche Forschungsgemeinschaft (KO 4687/1-1; postdoctoral fellowship to M.K.). The Kempe foundation is acknowledged for support to the NMR and X-ray crystallography infrastructure at Umeå University. The Knut and Alice Wallenberg Foundation is acknowledged for funding of the NMR infrastructure (NMR4life). The Umeå Protein Expertise Platform is acknowledged for providing reagents for protein overexpression.

\section{Author contributions}

M.K. and M.W.-W. designed research; M.K., J.Å., C.G. and U.H.S. performed experiments; M.K., M.W.-W., U.H.S. and A.E.S.-E. analysed data; and M.K. and M.W.-W. wrote the manuscript.

\section{Additional information}

Accession codes: The X-ray structures of AdK Y171W-apo, AdK P177A-apo, AdK Y171W-holo and AdK P177A-holo have been deposited to the Protein Data Bank under accession codes $4 \mathrm{X} 8 \mathrm{M}, 4 \mathrm{X} 8 \mathrm{H}, 4 \mathrm{X} 8 \mathrm{O}$ and $4 \mathrm{X} 8 \mathrm{~L}$, respectively. The assigned NMR chemical shifts have been deposited within the BMRB database for P177A-apo (accession number 25353), Y171W-apo (accession number 25357), P177A-holo (accession number 25360), Y171W-holo data set a (accession number 25361) and Y171W-holo dataset b (accession number 25362).

Supplementary Information accompanies this paper at http://www.nature.com/ naturecommunications

Competing financial interests: The authors declare no competing financial interests.

Reprints and permission information is available online at http://npg.nature.com/ reprintsandpermissions/

How to cite this article: Kovermann, M. et al. Structural basis for catalytically restrictive dynamics of a high-energy enzyme state. Nat. Commun. 6:7644 doi: 10.1038/ncomms8644 (2015).

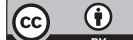

This work is licensed under a Creative Commons Attribution 4.0 International License. The images or other third party material in this article are included in the article's Creative Commons license, unless indicated otherwise in the credit line; if the material is not included under the Creative Commons license, users will need to obtain permission from the license holder to reproduce the material. To view a copy of this license, visit http://creativecommons.org/licenses/by/4.0/ 